\title{
Mania in late life
}

\section{Felicity Richards \& Martin Curtice}

\begin{abstract}
SUMMARY
Mania in late life is a serious disorder that demands specialist assessment and management. However, it is greatly under-researched, with only a paucity of studies specifically analysing older populations. The mainstay of the old age psychiatry workload will inevitably be concerned with assessing and managing dementia and depression, but the steady rise in the aging population with longer survival means that there will be an increase in absolute numbers of older people presenting with mania. There are no specific treatment algorithms available for mania in late life. This article reviews mania and hypomania in late life and concentrates on diagnosis, assessment and treatment, as well as on the management considerations associated with this important age group.
\end{abstract}

\section{DECLARATION OF INTEREST}

None.

Few studies have addressed the specific difficulties faced by old age psychiatrists when a patient presents with mania or hypomania in late life. The numbers of patients with atypical and typical presentations of mania in old age are not insignificant and with the steady rise of the aging population, coupled with better healthcare and greater survival, there will be increasing demands on service provision. Along with diagnostic uncertainties at presentation, mania in older adults tends to be more debilitating and severe than in the younger population, resulting in more frequent hospital admissions (Brooks 2005), higher rates of psychotic symptoms (Depp 2004) and higher levels of comorbidity and polypharmacy, with no specific treatment algorithms available to address these management considerations in older adults.

The literature on mania and hypomania in late life (defined as over 65 years of age) is scant and research papers tend to use different age cut-offs, preventing easy transfer of practical information. Nevertheless, we aim here to give an overview of mania and hypomania in this important age group.

\section{Classification of bipolar disorder}

There are two ways available to clinicians to classify episodes of bipolar disorder. Both ICD-10 (World Health Organization 1992) and DSM-IV (American Psychiatric Association 1994) allow for diagnoses of mania (with or without psychotic symptoms) and hypomania. It is unusual for an episode of mania or hypomania to occur in isolation.

According to ICD-10, for a diagnosis of bipolar disorder to be made, at least two episodes in which the patient's mood and activity levels are significantly disturbed (either with depression or mania) must be present. ICD-10 also characterises whether the current episode of bipolar affective disorder is mania or depression, and these are further divided into whether psychotic features are present or not.

DSM-IV classifies episodes into bipolar I and bipolar II disorders. Bipolar I disorder involves one or more manic or mixed episode. Individuals often have one or more major depressive episode. Bipolar II disorder is characterised by one or more major depressive episode, accompanied by at least one hypomanic episode.

\section{Terms specific to mania in late life}

Mania or hypomania may persist into late life as part of a lifelong affective illness, with these patients 'graduating' from the general adult services. Although mania usually presents before the age of 30 , with a further peak in females in their 50s, it can present for the first time in old age, with a third peak (especially in males) in the eighth and ninth decades (Depp 2004).

Many patients are believed to convert to mania in later life, commonly following recurrent depression. This can either be part of the natural progression of illness or be due to antidepressant-induced mania. It has been proposed that treatment with tricyclic antidepressants is twice as likely to result in a manic episode compared with treatment with selective serotonin reuptake inhibitors or placebo. Venlafaxine and duloxetine have also been found to increase the risk of a switch from depression into mania (Goodwin 2009).

Other useful concepts and subtypes have been put forward for use, especially in older adults (Box 1), including 'secondary mania' (or 'disinhibition syndrome') and 'vascular mania'.

\section{Secondary mania}

It is accepted that patients with forms of neurological illness can present with affective disorders. The concept of secondary mania, first described by Krauthammer \& Klerman (1978), was used to
Felicity Richards is a consultant in old age psychiatry at Worcestershire Mental Health Partnership NHS Trust, UK. She has a particular interest in affective disorders in the elderly. Martin Curtice is a consultant in old age psychiatry. He has a Master of Laws with Distinction in Mental Health Law and has an interest in mental health law and the Human Rights Act and affective disorders in the older person.

Correspondence Dr Felicity Richards, Department of Older Adult Psychiatry, Lucy Baldwin Unit, F Block, Kidderminster Hospital Bewdley Road, Kidderminster DY11 6RJ, UK. Email: felicity. richards1@worcsmhp.nhs.uk 
BOX 1 Summary of distinct subtypes

Early-onset bipolar disorder

Patients with bipolar disorder already known to general adult services who graduate into older age services and present with mania in later life

Latent bipolar disorder

Onset of depression at a younger age, converting to mania in later life, either owing to the natural progression of illness or a switch due to antidepressant medications, brain pathology, etc.

Secondary mania (disinhibition syndromes)

No previous history of affective disorders, with mania developing in association with a medical or neurological disorder. Can also include vascular mania and complicated mania

'Unipolar' mania as per DSM-IV (American Psychiatric Association 1994)

Mania in later life occurring in isolation, with none of the above associations

(Shulman 1999)

explain late-onset presentations of mania associated with a diverse group of medical conditions. Neurologists tend to favour the term 'disinhibition syndrome', but the clinical presentation and features are similar.

Secondary manias can be seen in patients with multiple sclerosis, Parkinson's disease, temporal lobe epilepsy, AIDS, dementia and traumatic brain injury (Schneck 2002). Indeed, secondary mania is seen in $9 \%$ of patients with traumatic brain injury, with a preponderance of basal temporal lesions noted in these patients. A positive family history of affective disorder and subcortical atrophy before injury are added risk factors (Shulman 2008).

A number of further studies have supported the concept of an association with neurological comorbidity. Tohen et al (1994) found that patients with a first-episode mania in old age were twice as likely to have a comorbid neurological disorder compared with those who had experienced multiple episodes. Many studies have suggested that secondary mania/disinhibition syndromes are associated with right-sided lesions, based on the hypothesis that disrupting connections within the orbitofrontal circuit mediates manic symptoms (Shulman 2008).

Other examples highlighting the heterogeneous nature of the causes of secondary mania include metabolic disturbance, endocrine disorders, such as thyrotoxicosis and hydrocortisone replacement, neoplasia and infection, all of which can also present as a delirium, often adding to diagnostic uncertainty (Shulman 2008)

\section{Vascular mania}

Steffans \& Krishnan (1998) proposed vascular mania as a subtype of mania. The concept is similar to that of 'vascular depression' proposed by Alexopoulous et al (1997) and is used to explain the high rates of manic symptoms seen in those who have evidence of cerebrovascular disease. This concept is supported by the relatively acute onset of manic symptoms that can occur following a cerebrovascular event, the higher prevalence of silent cerebral infarcts on neuroimaging in late-onset patients and the association of hyperintensities with risk factors such as hypertension, heart disease and diabetes (Shulman 2008; Tamashiro 2008).

\section{Epidemiology}

There appear to be discrepancies between the reported occurrence of bipolar disorder in the older adult population, and no clear consensus on mania/hypomania. A number of studies have consistently reported that the prevalence of bipolar disorder has an inverse relationship with age, with a decline in prevalence with increasing age (McCrone 2008; Byers 2010). Hirschfeld et al (2003) suggested that up to $0.5 \%$ of people over 65 will have bipolar disorder.

A 1-year incidence rate of $0.1 \%$ among adults over 65 has been indicated. This is lower than that for adults aged 45-65 (0.4\%) and 18-44 (1.4\%) (Depp 2004).

\section{Burden of bipolar disorder in older people}

\section{Economic}

Elderly patients can have a disproportionate impact on health services and some estimate that bipolar disorder accounts for approximately $8-10 \%$ of admissions to psychiatric units, similar to that of younger adults (Depp 2004). Research predicts increasing numbers of patients accessing services. A report by the King's Fund (McCrone 2008) estimated the projected greatest proportional increase in the number of people with bipolar disorder and related conditions is still in those aged 65 and over. However, the increase in the number of elderly people will not significantly affect the total number of new patients because of the lower prevalence in this group.

In the UK, the mean service costs of bipolar disorder and related conditions for those aged 65 and over are projected to be approximately four times greater than for younger age groups, owing to higher average in-patient and residential care costs (McCrone 2008). In this study, the elements used to estimate total costs were prescribed drugs, in-patient care, other National Health Service 
(NHS) services, supported accommodation, day care, other social services, informal care and lost employment.

Among older people, the rate of hospital admission for bipolar disorder is the same as that for schizophrenia, although the length of stay is shorter (Depp 2004). Compared with older outpatients with unipolar disorder, those with bipolar disorder use four times the amount of mental health services, including in-patient hospitalisation, case management, skills training and day hospitals (Bartels 2000). The overall cost of annual care of older adults with bipolar disorder and comorbid dementia are more than twice that for those with bipolar disorder alone (Sajatovic 2006).

\section{Morbidity and mortality}

Bipolar disorders are associated with higher morbidity and mortality. The increase in the number of comorbid medical conditions is directly proportional to age, and approximately $67 \%$ of over-70-year-olds who have bipolar have at least one significant medical condition (Beyer 2005). Patients with bipolar disorder, especially bipolar I, have increased mortality from cardiovascular causes in particular. The difference in cardiovascular mortality risk may reflect the physical health consequences of mania/hypomania because depressive symptom burden is not related to cardiovascular mortality (Fiedorowicz 2009).

An increase in morbidity from obesity and type 2 diabetes mellitus has been reported in people with bipolar disorder compared with the general population. Explanations for these possible increased risks include comorbidity with substance misuse and other medical conditions, inadequate prevention of cardiovascular risk factors (such as smoking, obesity and lack of exercise), the side-effects of psychotropic medications and poor engagement with general medical care (Morriss 2005).

\section{Suicide}

Bipolar disorder is associated with a high suicide risk (lifetime risk of 8-20\%; Aizenberg 2006), especially during depressive episodes or mixed states (Goodwin 2009). However, there is a lack of literature specifically concerning completed and attempted suicide in those over 65 with mania or hypomania. Factors for completed suicide in working-age patients that can be extrapolated to the elderly include inadequate treatment (medication) and inadequate follow-up by mental health services (Keks 2009). With the above in mind, elderly patients with bipolar disorder should be recognised as high risk and be provided with long-term intensive support (Goodwin 2009).

\section{Aetiology}

A family history of affective illness appears to increase the risk of bipolar disorder at any age. However, older patients with first-onset mania appear to have fewer first-degree relatives with affective illness, compared with patients presenting earlier. Figures are inconclusive, with results ranging from $24 \%$ to $88 \%$ for the presence of a positive family history (Shulman 2008).

\section{Diagnosis and assessment}

In the diagnosis of mania in late life, clinicians commonly use either the ICD-10 or the DSM-IV classification. However, it is important to note that rather than the classic symptoms of elevated mood and grandiosity, mania in the elderly can cause initial diagnostic uncertainty, with irritability, distractibility and disorientation being prominent presenting symptoms (Kennedy 2008), masking delirium, cognitive impairment or indeed depression. The differentiation between delirium and mania or hypomania can be aided by observation over time (Brooks 2005). Mania, therefore, should be included in the differential diagnosis of all elderly patients with a relatively acute onset of agitation and confusion, despite lack of a previous history of affective disturbance (Box 2).

Taking a comprehensive history, ideally with a collateral account, is invaluable. For a first presentation of mania, the history may reveal past episodes of hypomania or depression, not severe enough to warrant referral, treatment or intervention by mental health services. Classification of mania in later life will be helpful in deciding on management and future care (see Box 1 for mania subtypes).

Full investigations should include a complete physical examination, including neurological examination (Box 3). Routine blood tests should be carried out and an electrocardiogram taken, especially if lithium or valproate are being considered.

\section{B0X 2 Differential diagnosis of mania in late} life

- Delirium - hyperactive type

- Dementia - especially with frontotemporal involvement

- Stroke

- Early-onset mania, as part of coexisting bipolar affective disorder

- Secondary mania, i.e. due to medications, physical illness

- Late-onset schizophrenia-like psychosis

- Acute and transient psychotic disorders 
BOX 3 Investigations for mania in late life

- Physical examination, including neurological examination

- Blood investigations - urea and electrolytes, glomerular filtration rate, liver function tests, thyroid function tests, full blood count, vitamin $B_{12}$ and folate, bone profile, glucose

- Midstream specimen of urine

- Electrocardiogram (ECG)

- Chest X-ray

- Computed tomography (CT) and/or magnetic resonance imaging (MRI) if indicated (in the presence of neurological signs, etc.)

Full exploration of any causes of delirium should be excluded, including a midstream specimen of urine. Mania in older adults is associated with a high rate of medical and neurological disease (Tohen 1994). Consequently, all patients presenting with first-onset mania should be carefully screened for contributing medical disorders and brain imaging is useful in this context.

Owing to the known associations with bipolar disorder and cardiovascular risk, initial assessment should also include careful history of common cardiovascular risk factors, such as smoking, excessive alcohol use, hypertension, hypercholesterolaemia and diabetes (Shulman 2008).

With the consideration of the causes of secondary mania discussed above, medication(s) should be examined and any recent changes identified - caused by associations with antidepressants, antibiotics ('antibiomania'), in particular clarithromycin and other macrolides, steroids and oestrogens - all examples of inducing agents for secondary mania (Abovesh 2002; Kennedy 2008).

\section{Screening instruments}

The diagnosis of mania relies on clinical evidence and assessment. In clinical practice there are no screening tools available for easy use in psychiatric or general hospital settings. Diagnostic scales can aid in clinical diagnosis, but are mainly used in the research setting (Scottish Intercollegiate Guidelines Network 2005). Self-report questionnaires may be more difficult to use with elderly people because of sensory impairments.

\section{Psychiatric comorbidity}

\section{Anxiety disorders and substance misuse}

Psychiatric comorbidity in bipolar disorder is widely documented. Reports have indicated that generalised anxiety disorder shows a lifetime prevalence of
$20.5 \%$ and a 12 -month prevalence of $9.5 \%$ in older patients with bipolar disorder. For panic disorder, lifetime prevalence is as high as $19 \%$, whereas 12-month prevalence is $11.9 \%$ (Goldstein 2006). Similarly, comorbid post-traumatic stress disorder, substance misuse, 'other anxiety' or dementia have been found to occur in nearly $29 \%$ of older adults with bipolar disorder (Sajatovic 2006).

Substance misuse, a significant comorbidity in younger adults with bipolar disorder, is less common in older cohorts, but nevertheless occurs more frequently than in healthy elderly controls. A lifetime and 20-month prevalence of 38\% for comorbid alcohol use disorders has been reported (Goldstein 2006), and patients with bipolar disorder with a lifetime history of substance misuse appear to have a greater number of hospital admissions (Depp 2004).

\section{Cognitive impairment}

Cognitive impairment in late-onset bipolar disorder has been widely reported and is associated with more severe cognitive impairment than in early-onset disorder (Schouws 2009). Compared with age-matched controls, patients with bipolar disorder score lower on most cognitive measures, with patients late-onset disorder showing more impairment in psychomotor performance and mental flexibility. Older adults whose onset of bipolar illness had been before the age of 50 were found to have impairment across a range of domains, including selective attention, verbal memory and verbal fluency, in the euthymic state. It has been concluded that older patients with bipolar disorder may have substantial cognitive impairments, perhaps indicating a trait-like cognitive disability related to the disease (Schouws 2007).

\section{Management}

Management of mania or hypomania in late life is complex. Onset is often relatively sudden and severe and individuals will therefore most likely need in-patient admission, either as informal patients or under the Mental Health Act. Before and following admission, the importance of good working and understanding of this disorder within multidisciplinary teams is vital. The recognition and management of concurrent physical illnesses is as much the job of the old age psychiatrist as of the general practitioner.

It is not uncommon for patients to have poor engagement with primary care and contact with psychiatric services can often aid in screening for physical disorders. Issues such as adherence and the practicalities of medication prescribing in this age group need to be addressed, as well as an awareness 
of polypharmacy and potential drug-drug interactions with a number of the psychotropics discussed below. Care planning should consider the patient's social situation and the effects that the illness can have on family members and carers.

\section{Pharmacological management}

Treatment of older people often involves compromise because the side-effects of treatment may be as harmful as the condition being treated. There are no specific treatment algorithms for mania in late life, with a scarcity of published controlled trials in the elderly. In practice, treatment for older adults with mania/hypomania generally follows similar guidelines as for other groups. However, certain precautions should be taken because of the differences in pharmacokinetics, side-effects, concomitant medication use and comorbidity. Adherence at times can be difficult, because of the nature of acute episodes, the presence of cognitive impairment or a reluctance to take medication.

As a general guide, medication doses are lower and should be titrated with care, owing to reduced volume of distribution and reduced renal clearance - especially important if thinking about lithium initiation. Pharmacotherapy can be divided into different phases of treatment: acute (manic or mixed episodes) and maintenance treatment. Comprehensive guidance is available from the British Association of Psychopharmacology (Goodwin 2009) and the National Institute for Health and Clinical Excellence (2006; Box 4).

\section{Lithium}

There are no trials looking at the use of lithium treatment in late-life bipolar disorder, either for acute episodes or as maintenance therapy. However, lithium continues to be used commonly as a mood stabiliser in older adults (Shulman 2003). Advanced age, absence of a family history of bipolar disorder, mania secondary to another medical condition (e.g. stroke) or dementia predict poor response to lithium (Kennedy 2008). Of note, lithium treatment may protect elderly patients with bipolar disorder against Alzheimer's disease (Nunes 2007) and, with regard to longer-term treatment, lithium specifically is associated with a reduced risk of suicide (Aizenberg 2006; Goodwin 2009).

The impact of age-related decline in renal function is an important consideration. Renal function during chronic lithium treatment is related to age, lithium intoxication episodes, pre-existing renal disease and treatment schedule, rather than to duration of prophylactic lithium therapy; with lithium use for more than 10 years, 10-20\% of patients may display morphological kidney changes, not generally associated with kidney failure (Hetmar 1991; Goodwin 2009).

The pharmacokinetics of lithium show that the rate of excretion in older people is approximately half that in the younger population and, because of this, many older people respond to much lower doses. A practice guideline is available for lithium use when incidental chronic kidney disease is detected in those in whom lithium is being considered or when long-term lithium users develop chronic kidney disease (Kripalani 2009).

Adverse effects of lithium are not confined to the kidney and they encompass a range of systemic complaints. Lithium-induced tremor, aggravation of Parkinsonian tremor and spontaneous extrapyramidal symptoms can occur (Shulman 2008). Mild tremor and nystagmus can present without functional consequences and should not be considered signs of toxicity (Kennedy 2008).

Signs of lithium toxicity include gastrointestinal complaints, ataxia, slurred speech, delirium or coma. They may follow periods of dehydration due to vomiting, diarrhoea or inadequate fluid intake, or (especially pertinent in the elderly) periods of immobility, development of chest infections or sepsis (National Institute for Health and Clinical Excellence 2006). Higher rates of hypothyroidism have been found in elderly people on lithium and will need appropriate treatment.

Drug interactions are an important consideration. Concomitant use of medications that lower renal clearance can potentially increase the risk of lithium toxicity. Clinicians should always be aware of medications such as thiazide diuretics, angiotensin-converting enzyme (ACE) inhibitors and non-steroidal anti-inflammatory medications, and monitor the blood more frequently in these patients (National Institute for Health and Clinical Excellence 2006).

\section{BOX 4 NICE recommendations for treating bipolar disorder in older people}

When treating older people with bipolar disorder, healthcare professionals should:

- be aware of the need to use medication at lower doses

- be alert to the increased risk of drug interactions when prescribing psychotropic medication to older adults

- ensure that medical comorbidities have been recognised and addressed

- be aware that older people are at greater risk of sudden onset of depressive symptoms after recovery from a manic episode

(National Institute for Health and Clinical Excellence 2006) 


\section{Valproate}

Valproate has been shown to be effective and well tolerated in older people, although there is no current evidence that it is any better than lithium in treating bipolar disorders (Shulman 2003). A positive response to valproate has been correlated to older age, increased severity of manic symptoms, neurological impairment, dysphoria and a history of lithium non-responsiveness (Sajatovic 2002).

When prescribing valproate, clinicians should be aware of its interactions with other anticonvulsants, and the need for more careful monitoring of sedation, tremor and gait disturbance in older people. Fully reversible cognitive impairment and Parkinsonism caused by valproate have been reported with the use of sodium valproate (Walstra 1997; Schreur 2009). The British Association for Psychopharmacology (Goodwin 2009) recommends starting doses of $500 \mathrm{mg}$ daily in older people. However, starting doses of $125-250 \mathrm{mg}$ at night, titrating carefully up to a maintenance dose of 500-1000 mg/day has also been recommended (Sajatovic 2002).

\section{Carbamazepine}

Carbamazepine's use as a first-line treatment is not encouraged (Goodwin 2009), but it may be the agent of choice for secondary mania because elderly patients with this diagnosis have been found to respond relatively poorly to lithium (Depp 2004). Particularly concerning with carbamazepine is the risk of drug-drug interactions due to its induction of the P450 enzyme system. Consequently, the efficacy of medications such as antipsychotics and antidepressants can be affected, along with commonly prescribed drugs such as calcium channel blockers, erythromycin and warfarin.

In elderly patients, serum electrolytes should be routinely checked because of the increased potential for inducing hyponatraemia (Goodwin 2009), and special attention should be paid to a history of blood dyscrasias or liver disease. Side-effects are wide-ranging and include ataxia, confusion, diplopia and blurred vision, as well as fatigue, gastrointestinal problems and rare idiosyncratic effects such as Stevens-Johnson syndrome, agranulocytosis and hepatic failure.

\section{Antipsychotics}

Mania in late life can develop quickly, potentially putting the patient and others at risk. For more severe forms, especially mania with psychotic symptoms, treatment with antipsychotics is at times unavoidable for initial stabilisation (Goodwin 2009). For patients who present with severe mania when already taking lithium or valproate, adding an antipsychotic should be considered while gradually increasing the dose of lithium or valproate (National Institute for Health and Clinical Excellence 2006; Goodwin 2009). If treating mania with antipsychotics, olanzapine, quetiapine or risperidone would normally be used.

Generally, patients with bipolar disorder are more likely to develop extrapyramidal side-effects than patients with schizophrenia on comparable doses of antipsychotics (Goodwin 2009). Elderly patients with primary bipolar disorder have been found to have high rates of tardive dyskinesia (approaching $20 \%$ ), with the prevalence increasing with age (Sajatovic 2002). There has been conflicting and inconclusive evidence that antipsychotics may increase the rate of cognitive decline in patients with dementia (Livingston 2007).

\section{Non-pharmacological management \\ Electroconvulsive therapy}

Electroconvulsive therapy may be indicated to gain rapid control and short-term improvement in a patient who is severely unwell. This is generally after all other treatment options have been considered and have failed, or when the situation is thought to be life-threatening (National Institute for Health and Clinical Excellence 2003). Examples could include complications of self-neglect, dehydration or morbid nutritional status, suicidal risk or poor medication efficacy or tolerance. Electroconvulsive therapy should be considered based on an assessment of risks and benefits. Especially in the elderly, anaesthetic risk, comorbidity and potential cognitive impairment/postictal confusion should be explored in detail. Care should be taken to assess consent after discussions of the risks and benefits with the patient and, where appropriate, their advocate. It would be likely that the Mental Health Act would need to be implemented in such clinical scenarios where the patient lacked the requisite capacity to consent. Any advanced decision to refuse treatment should be consulted.

\section{Psychotherapy}

Psychotherapy is a valuable element in the management of bipolar disorder in general, but mainly for depressive symptoms. There is no evidence for the treatment of refractory disease with psychotherapy in the older adult population. Whereas most, if not all, advice pertains to those under 65 , the key areas for psychotherapy in bipolar disorder that could be pertinent to older people are discussed below.

\section{Psychoeducation}

For mania, psychoeducation has been suggested to help individuals identify signs of relapse (relapse signature), to improve adherence to treatment 
(adherence/compliance therapy) and to enhance general coping strategies. The maintenance of regular behavioural patterns, such as a person's daily routine and sleep, appear to be important considerations in the elderly (National Institute for Health and Clinical Excellence 2006).

\section{Cognitive-behavioural therapy}

Cognitive-behavioural therapy has been found to reduce the likelihood of isolated manic symptoms progressing into a full episode.

\section{Other types of psychotherapy}

In the general adult population, family-focused therapy, group treatments (such as group psychoeducation as an adjunct to pharmacotherapy), interpersonal and social rhythm therapy all appear to have a role (Goodwin 2009). Their use in the older population still needs to be reviewed and other considerations such as cognitive functioning, motivation and sensory impairment may affect the potential efficacy in this population. The Department of Health (2009) has stated that service provision should be equal among all ages. Therefore, one would expect availability and access to the same type of psychotherapy as for younger adults.

\section{The course of mania in late life}

Literature on the course of mania in late life is limited. From experience, patients presenting to the older adult wards appear to do just as well as younger adults in the acute phase of illness, achieving similar outcomes. However, it has been noted that overall outcome is generally worse in mania than in depression, with a higher prevalence of persistence of symptoms, cognitive decline and greater mortality (Shulman 1999).

\section{Prevention}

There is no solid evidence base for prevention of mania in late life. Primary prevention would be designed to look at influencing modifiable factors. The higher cerebrovascular and cardiovascular risk in many elderly patients with mania has significant management implications and would warrant close monitoring and treatment of potential risk factors, in both primary and secondary care. Treatment and maintenance care for chronic illnesses and physical disability, as well as an awareness of polypharmacy and potential drug interactions, are important.

Secondary prevention involves reducing the risk of relapse of illness. For younger age groups, it is recommended that a mood stabiliser be prescribed as prophylaxis after a single manic episode, if associated with significant risk and adverse consequences, and that treatment should continue for at least 2 years, or longer in highrisk patients (National Institute for Health and Clinical Excellence 2006). Clinicians working with older adults should monitor treatment closely and may have to use their own judgement on the risks $v$. the benefits of treatment. Other strategies for preventing relapse may involve techniques such as cognitive-behavioural therapy, psychotherapy or social rhythms therapy. Social inclusion, community support and adherence to medication may further help to reduce future recurrences of mania.

\section{Discussion}

There is a paucity of evidence on the treatment and management of mania in late life. This review builds on the central tenets of any comprehensive older adult psychiatric assessment process, in particular providing detailed information on diagnostic and treatment issues. It is also important to consider patient and carer education. The Royal College of Psychiatrists (2007) and Patient UK (2009) provide helpful information for older patients and their carers.

The preponderance of work in old age psychiatry will inevitably involve dementia and depression, but specialist knowledge in the assessment and nuances of management of mania in older adults is equally important. This fits perfectly with the 'right help, right time' concept advocated in the New Horizons programme (Department of Health 2009).

\section{References}

Abovesh A, Stone C, Hobbs WR (2002) Antimicrobial induced mania (antibiomania). A review of spontaneous reports. Journal of Clinical Psychopharmacology 22: 71-81.

Aizenberg D, Olmer A, Barak Y (2006) Suicide attempts amongst elderly bipolar patients. Journal of Affective Disorders 91: 91-4.

Alexopoulous GS, Meyers BS, Young RC, et al (1997) Clinically defined vascular depression. American Journal of Psychiatry 154: 562-5.

American Psychiatric Association (1994) Diagnostic and Statistical Manual of Mental Disorders (4th edn) (DSM-IV). APA.

Bartels S, Forester B, Miles K, et al (2000) Mental health service use by elderly patients with bipolar disorder and unipolar major depression. American Journal of Geriatric Psychiatry 8: 160-6.

Beyer J, Kuchibhatla M, Gersing K, et al (2005) Medical comorbidity in a bipolar outpatient clinical population. Neuropsychopharmacology 30: $401-4$

Brooks J0, Hoblyn JC (2005) Secondary mania in older adults. American Journal of Psychiatry 162: 2033-8.

Byers AL, Yaffe K, Covinsky KE, et al (2010) High occurrence of mood and anxiety disorders among older adults. Archives of General Psychiatry 67: 489-96.

Department of Health (2009) New Horizons: A Shared Vision for Mental Health. Department of Health (http://www.dh.gov.uk/en/Publicationsand statistics/Publications/PublicationsPolicyAndGuidance/DH_109705).

Depp CA, Jeste DV (2004) Bipolar disorder in older adults. A critical review. Bipolar Disorders 6: 343-67.

Fiedorowicz JD, Solomon DA, Endicott J, et al (2009) Manic/hypomanic symptom burden and cardiovascular mortality in bipolar disorder. Psychosomatic Medicine 71: 598-606.
MCO answers

$1 d \quad 2$ b 3 c 4 c 5 e 
Goldstein BI, Herrmann N, Shulman KI (2006) Comorbidity in bipolar disorder among the elderly. Results from an epidemiological community sample. American Journal of Psychiatry 163: 319-21.

Goodwin GM (2009) Evidence-based guidelines for treating bipolar disorder. Revised second edition. Recommendations from the British Association for Psychopharmacology. Journal of Psychopharmacology 23: $346-88$.

Hetmar 0, Povlsen UJ, Ladefoged J, et al (1991) Lithium: long-term effects on the kidney. A prospective follow-up study ten years after kidney biopsy. British Journal of Psychiatry 158: 53-8.

Hirschfeld RM, Calabrese JR, Weissman MM, et al (2003) Screening for bipolar disorder in the community. Journal of Clinical Psychiatry 64: 53-9.

Keks NA, Hill C, Sundram S, et al (2009) Evaluation of treatment in 35 cases of bipolar suicide. Australian and New Zealand Journal of Psychiatry 43: 503-8.

Kennedy GJ (2008) Bipolar disorder in late life. Mania. Primary Psychiatry 15: 28-33.

Krauthammer C, Klerman GL (1978) Secondary mania. Manic syndromes associated with antecedent physical illness or drugs. Archives of General Psychiatry 35: 1333-9.

Kripalani M, Shawcross J, Reilly J, et al (2009) Lithium and chronic kidney disease. BMJ 339: 166-70

Livingston G, Walker AE, Katona CLE, et al (2007) Antipsychotics and cognitive decline in Alzheimer's disease. The LASER-Alzheimer's disease longitudinal study. Journal of Neurology, Neurosurgery and Psychiatry 78: $25-9$.

McCrone P, Dhanasiri S, Patel A, et al (2008) Paying the Price. The Cost of Mental Health Care in England to 2026. King's Fund.

Morriss R (2005) Metabolism, lifestyle and bipolar affective disorder. Journal of Psychopharmacology 19: 94-101

National Institute for Health and Clinical Excellence (2003) Electroconvulsive Therapy (ECT) Guidance. TA59. NICE (http://www. nice.org.uk/TA059)

National Institute for Health and Clinical Excellence (2006) Bipola Disorder. The Management of Bipolar Disorder in Adults, Children and Adolescents, in Primary and Secondary Care. NICE Clinical Guideline 38. NICE (http://www.nice.org.uk/nicemedia/live/10990/30193/30193.pdf).

Nunes PV, Forlenza OV, Gattaz WF (2007) Lithium and risk for Alzheimer's disease in elderly patients with bipolar disorder. British Journal of Psychiatry 190: 359-60

Patient UK (2009) Bipolar Disorder. Patient Information Publications (http://www.patient.co.uk/health/Bipolar-Affective-Disorder.htm).
Royal College of Psychiatrists (2007) Bipolar Disorder (Manic Depression). RCPsych (http://www.rcpsych.ac.uk/mentalhealthinfoforall/problems/ bipolarmanicdepression/bipolardisorder.aspx).

Sajatovic M (2002) Treatment of bipolar disorder in older adults. International Journal of Geriatric Psychiatry 17: 865-73.

Sajatovic M, Blow FC, Ignacio RV (2006) Psychiatric comorbidity in older adults with bipolar disorder. International Journal of Geriatric Psychiatry 21: $582-7$

Schneck CD (2002) Bipolar disorder in neurologic illness. Current Treatment Options in Neurology 4: 477-86.

Schouws SN, Zoeteman JB, Comijs HC, et al (2007) Cognitive functioning in elderly patients with early onset bipolar disorder. International Journal of Geriatric Psychiatry 22: 856-61.

Schouws SNTM, Comijs HC, Stek ML, et al (2009) Cognitive impairment in early and late bipolar disorder. American Journal of Geriatric Psychiatry 17: $508-15$.

Schreur L, Middeljans-Tijssen C, Hengstman G, et al (2009) Cognitive impairment and parkinsonism due to the use of sodium valproate. Tijdschrift voor Gerontologie en Geriatrie 40: 29-33.

Scottish Intercollegiate Guidelines Network (2005) Bipolar Affective Disorder. A National Clinical Guideline. SIGN (http://www.sign.ac.uk/ $\mathrm{pdf} /$ sign82.pdf)

Shulman Kl, Herrmann N (1999) The nature and management of mania in old age. Psychiatric Clinics of North America 22: 649-65.

Shulman Kl, Herrmann N (2008) Mood disorders. Manic syndromes in old age. In The Oxford Textbook of Old Age Psychiatry (eds R Jacoby, C Oppenheimer, T Dening, et al): 557-62. Oxford University Press.

Shulman KI, Rochon P, Sykora K, et al (2003) Changing prescription patterns for lithium and valproic acid in old age. Shifting practice without evidence. BMJ 326: 960-1.

Steffans DC, Krishnan KRR (1998) Structural neuroimaging and mood disorders. Recent findings, implications for classification, and future directions. Biological Psychiatry 43: 705-12

Tamashiro JH, Zung S, Zanetti MV, et al (2008) Increased rates of white matter hyperintensities in late-onset bipolar disorder. Bipolar Disorders 10: 765-75.

Tohen M, Shulman KI, Satlin A (1994) First episode mania in late life. American Journal of Psychiatry 151: 130-2.

Walstra G (1997) Reversible dementia due to valproic acid therapy. Nederlands Tijdschrift voor Geneeskunde 141: 391-3.

World Health Organization (1992) The ICD-10 Classification of Diseases, Clinical Descriptions and Diagnostic Guidelines. WHO.

\section{MCOs}

Select the single best option for each question stem

1 Epidemiological studies have shown:

a the 1-year prevalence of bipolar disorder is $1 \%$ in the over $65 \mathrm{~s}$

$\mathrm{b}$ the prevalence of bipolar disorder among community-based samples increases in later life

c in women, the incidence of bipolar disorder may rise in the eighth and ninth decade

$d$ the increased number of older adults will significantly affect service provision for mania in late life

e up to $1 \%$ of psychiatric admissions for over 65 s at any given time are attributable to bipolar disorder.

\section{In older patients presenting with} secondary mania:

a a positive family history is not a risk factor b comorbid neurological disorders are twice as likely to be found in first episodes

c it is an unusual cause of mania

$\mathrm{d}$ a trend towards left-sided lesions has been confirmed

e clinical features are synonymous with complicated mania.

\section{In late-onset mania, compared with} early-onset mania:

a psychiatric comorbidity is rare

b a positive family history of affective disorders is found as frequently

c prominent presenting symptoms are disorientation, distractibility and irritability

d a fully comprehensive assessment of medical history and current medications is not as important

e imaging (MRI/CT) is rarely recommended.
4 Pharmacological management of mania in late life:

a includes specific treatment algorithms for use in over $65 \mathrm{~s}$

$\mathrm{b}$ is less effective than for younger patients

c generally includes lower starting doses, with careful titration

d commonly involves typical antipsychotics

$\mathrm{e}$ is less effective than psychological intervention in the acute phase of illness.

5 Lithium treatment in late-onset mania:

a should be used infrequently and with caution

b can cause cognitive impairment

c has a good response rate in those with dementia

$\mathrm{d}$ requires the same dose range as for younger adults

e has a lower response rate in those with secondary mania. 